\title{
Use of the Tego needlefree connector is associated with reduced incidence of catheter-related bloodstream infections in hemodialysis patients
}

This article was published in the following Dove Press journal: International Journal of Nephrology and Renovascular Disease 3 April 2014

Number of times this article has been viewed

\author{
Steven M Brunelli' \\ Levi Njord ${ }^{2}$ \\ Abigail E Hunt ${ }^{\prime}$ \\ Scott P Sibbel' \\ 'DaVita Clinical Research ${ }^{\circledR}$, \\ Minneapolis, MN, USA; ${ }^{2}$ DaVita \\ HealthCare Partners, Inc, Denver, \\ CO, USA
}

\begin{abstract}
Background and objectives: Catheter-related bloodstream infections (CRBSIs) are common in hemodialysis patients using central venous catheters, and catheter occlusion also occurs frequently. The Tego needlefree connector was developed to reduce the incidence of these complications; however, existing studies of its effectiveness and safety are limited.
\end{abstract}

Materials and methods: This retrospective analysis compared outcomes among patients of a large dialysis organization receiving in-center hemodialysis using a central venous catheter with either the Tego connector or standard catheter caps between October 1 and June 30, 2013. Incidence rates for intravenous (IV) antibiotic starts, receipt of an IV antibiotic course, positive blood cultures, mortality, and missed dialysis treatments were calculated, and incidence-rate ratios (IRRs) were estimated using Poisson regression models. Utilization of erythropoiesis-stimulating agents (ESAs) and thrombolytics was described for each patient-month and compared using mixed linear models. Models were run without adjustment, adjusted for covariates that were imbalanced between cohorts, or fully adjusted for all potential confounders.

Results: The analysis comprised 10,652 Tego patients and 6,493 controls. Tego use was independently associated with decreased risk of CRBSI, defined by initiation of IV antibiotics (adjusted IRR $0.92,95 \%$ confidence interval [CI] $0.87-0.97$ ) or initiation of IV antibiotic course (adjusted IRR 0.89 , 95\% CI $0.84-0.95$ ). Tego use was independently associated with decreased rate of missed dialysis treatments (adjusted IRR 0.98, 95\% CI 0.97-1.00); no significant difference between Tego and control cohorts was observed with respect to mortality. Tego use was associated with decreased likelihood of thrombolytic use (adjusted per-month probability of 5.6\% versus $6.2 \%$ for controls) and lower utilization of ESAs in study months 7-9.

Conclusion: Use of the Tego connector may reduce the risk of CRBSI and result in lower utilization of thrombolytics, antibiotics, and ESAs, as well as fewer missed dialysis treatments.

Keywords: catheter, dialysis, end-stage renal disease, ESA, infection, mortality

\section{Introduction}

Catheter-related bloodstream infections (CRBSIs) are a common complication of long-term use of central venous catheters (CVCs) for vascular access in end-stage renal disease (ESRD) patients receiving hemodialysis (HD). Data from the United States Renal Data System suggest that from 2000 to 2008, hospitalizations for bacteremia and sepsis increased, a change that parallels the increase in use of CVCs among US HD patients. From 2008 onward, rates have remained high: at approximately 300 admissions with bacteremia/sepsis per 1,000 patient-years in $2011 .{ }^{1}$ Aside from having a direct impact on patient well-being, catheter infection, including subclinical
Correspondence: Steven M Brunelli DaVita Clinical Research ${ }^{\circledR}, 825$ South 8th Street - Suite 300, Minneapolis, MN 55404, USA

Tel + I 78I $292090 \mid$

Fax + I $877683267 \mid$

Email steven.brunelli@davita.com 
colonization, also contributes to systemic inflammation, which has important downstream consequences, including increased dose requirements for erythropoiesis-stimulating agents (ESAs). ${ }^{2,3}$

Catheter occlusion due to thrombosis is another common issue, and can result in lower blood flow and dialysis inadequacy. ${ }^{4}$ To prevent catheter dysfunction caused by thrombotic occlusions, standard protocols require that CVCs are locked with heparin after completion of a treatment. ${ }^{5,6}$ However, a number of concerns have been raised regarding heparin use: dosing errors or overfilling of the catheter lumen can result in systemic anticoagulation and heparin-induced thrombocytopenia, and heparin has also been shown to promote Staphylococcus aureus biofilm formation in vitro. ${ }^{7,8}$

Recent initiatives to reduce rates of CVC use in HD patients have had some success,, 910 although rates remain unacceptably high. ${ }^{1}$ A variety of factors contribute to the high rates of CVC use in HD patients, including lack of predialysis care, maturation failure for arteriovenous fistulas and grafts, patient resistance to permanent access placement, and fistula stenosis and thrombosis. ${ }^{11}$ Therefore, despite concerted efforts to reduce catheter use, a proportion of HD patients will remain reliant on this access type.

The Tego ${ }^{\circledR}$ needlefree hemodialysis connector (ICU Medical, Inc., San Clemente, CA, USA) was developed to reduce catheter-related infections and thrombotic occlusions associated with the use of CVCs for HD. ${ }^{12}$ The Tego connector device has been cleared by the US Food and Drug Administration, and can be flushed and locked with either saline or heparin. Between November 2010 and July 2011, DaVita $^{\circledR}$ implemented use of the Tego connector in nearly $70 \%$ of its CVC patients. Implementation was not universal, owing to delayed or absent uptake by individual facilities. However, within individual facilities, Tego connectors are nearly universally used or universally not used. The resulting natural experiment forms the basis for rigorous evaluation of the comparative effectiveness of Tego versus standard catheter caps, an analysis that is lacking from the published literature; available studies have been small, and yielded equivocal results. ${ }^{13,14}$ The current retrospective, observational analysis was designed to assess the comparative effectiveness and safety of Tego connectors, relative to standard catheter caps, in a large, real-world, in-center HD patient population at a large dialysis organization (LDO) with respect to rates of catheter-related bacteremia, missed treatments, death, and utilization of ESAs and thrombolytics.

\section{Materials and methods}

\section{Study setting and patients}

The goal of this retrospective analysis was to assess the comparative effectiveness of Tego versus standard catheter caps. In order to minimize any bias due to events that preceded the study, analysis was limited to catheters that were newly placed during the study period. Although catheter implantation-site information was not available for study patients, tunneled catheters within the upper central vasculature are almost universal within LDOs. Patients were therefore considered if they: 1) underwent CVC placement during the period from October 1, 2012 to June 30, 2013; 2) received in-center HD via said CVC for at least 21 days thereafter (to avoid consideration of temporary catheters that may not have been left in place long enough to manifest adverse events); 3) had available electronic health-record data regarding the catheter-cap type used (ie, Tego or standard catheter cap); and 4) did not switch (in either direction) between standard catheter cap and Tego-connector use within 21 days of first in-center HD with the new catheter. CVCs in place prior to October 1, 2012 were not considered. However, patients with prior catheters were eligible for study if they underwent placement of a new catheter during the study period and met the aforementioned criteria; in these instances, patients entered the study at the time of the new catheter placement. Catheters with standard caps were typically locked with unfractionated heparin, and catheters with Tego connectors were locked with saline, as per LDO policies and procedures.

\section{Exposure}

Patients were classified according to the catheter cap type that was placed at the dialysis session following catheter placement (Tego versus standard catheter cap). By inclusionary restriction, the catheter type was maintained for at least 21 days thereafter (see earlier). Thereafter, patients remained assigned to their starting group for the remainder of the study. Patients who crossed over between exposure groups thereafter were censored 21 days after crossover; this lagged censoring was used to account for delays in disease manifestation, as infections may have initiated during use of the original cap type but not manifested until after switching. At-risk time otherwise continued until censoring for change in dialysis access type, transfer of care, change in modality, transplant, withdrawal from dialysis, or end of the study (June 30, 2013). Patients could therefore contribute a minimum of 21 days and a maximum of 270 days of at-risk time. 


\section{Outcome definitions}

Occurrence of CRBSI was identified for each patient using three operational definitions: intravenous (IV) antibiotic start, receipt of an IV antibiotic course (defined as patient having received two or more doses of IV antibiotic within a 21-day period), or confirmed positive blood culture. Blood cultures were grouped by a 21-day rule stipulating that any positive blood cultures were considered part of the same infection if the draw dates were $\leq 21$ days apart. Of particular note, many dialysis patients (particularly when faced with more severe symptoms) will seek urgent care outside the dialysis facility (eg, emergency department, hospital); blood culture results from such visits are not available through LDO electronic health records. Therefore, outcomes based on LDO records of positive blood cultures are insensitive measures of CRBSI, and were considered subordinate to CRBSI definitions based on IV antibiotic utilization, particularly initiation of antibiotic courses.

Death data were abstracted from the LDO electronic health record system. Missed treatments were also abstracted from LDO electronic health records. Missed treatment rate is important in its own right, because of implied effects on the adequacy of renal replacement therapy and also as a surrogate of hospitalization; sensitivity of LDO electronic health records for hospitalization events and particular cause-specific hospitalization events is poor. ESA and thrombolytic utilization were abstracted from LDOs' treatment records.

\section{Statistical analysis}

Incidence rates (death, hospitalization, missed dialysis sessions, CRBSI under each definition) were calculated as the number of events divided by time at risk. Incidence rate ratios (IRRs) were estimated using Poisson regression models.

Drug use or nonuse (for ESAs and thrombolytics) was described for each patient-month and compared using generalized estimating equations with logit link to a binomial distribution. ESA dose was considered among ESA users as the mean per-treatment dose for each patient-month and compared using mixed linear models. Tego exposure (dichotomous) was set as the primary fixed-effect term. Random patient intercepts were included, allowing for estimation of ESA dose at the patient level. Additional fixed-effect terms were entered as covariates in the mixed models. ESA dose was estimated on the log scale; results were back-transformed to the native scale.

All models were run to three specifications: unadjusted for any covariate, adjusted for potential confounders that were imbalanced between exposure groups (age, race, vintage, etiology of ESRD; model 1), and adjusted for all potential confounders considered (model 2). All data were analyzed using Stata 10.0 MP (StataCorp, College Station, TX, USA).

\section{Results}

Of 17,175 patients identified who met all inclusion criteria for the study, 29 patients who were $<18$ years old and one patient for whom information on sex was missing were excluded, leaving an analytical cohort of 17,145 patients. Of these, 10,652 patients (62.13\%) were classified as Tego catheter connecter users, and 6,493 patients $(37.87 \%)$ were classified as conventional catheter cap users.

\section{Patient characteristics}

Demographic information for the Tego and control cohorts at the time of first qualifying catheter placement in the study time frame is provided in Table 1 . The mean age of Tego patients was 61.7 years, and the mean age of control patients was 61.3 years. Tego patients were more likely to be Hispanic (17.5\% versus $13.3 \%)$ and less likely to be Black (31.6\% versus $33.5 \%)$ than controls. Differences between the cohorts in dialysis vintage and etiology of ESRD were observed: a higher proportion of Tego patients were new to dialysis ( $47.7 \%$ for Tego versus $41.9 \%$ for controls) and a lower proportion of Tego patients had been on dialysis for more than 12 months (32.4\% versus 37.6\%), while Tego patients were more likely to have ESRD attributed to hypertension (28.3\% versus $26.3 \%$ ) and less likely to have ESRD attributed to diabetes or other causes $(44.9 \%$ and $26.8 \%$ for Tego versus $45.9 \%$ and $27.8 \%$ for controls, respectively). There was no significant sex imbalance between Tego patients and controls ( $46.0 \%$ female for Tego patients versus $46.1 \%$ for controls) and there were no meaningful differences in Charlson Comorbidity Index score, a composite score that reflects the number and severity of comorbid conditions and which has been shown to predict patient 10 -year mortality, ${ }^{15}$ or the presence of specific comorbid conditions.

\section{CRBSIs}

Three metrics of CRBSIs were assessed: IV antibiotic starts, IV antibiotic courses (defined as receipt of two or more doses of IV antibiotics within a 21-day period), and positive blood cultures. The number of events, crude rates, and IRRs for each are reported in Table 2, and IRRs are represented graphically in Figure 1A. 
Table I Demographic comparisons for Tego and control cohorts

\begin{tabular}{|c|c|c|c|}
\hline & $\begin{array}{l}\text { Control } \\
(n=6,493)\end{array}$ & $\begin{array}{l}\text { Tego } \\
(n=10,652)\end{array}$ & $P$-value \\
\hline \multicolumn{3}{|l|}{ Age, years } & 0.11 \\
\hline Mean \pm SD & $61.3 \pm 15.2$ & $61.7 \pm 15.2$ & \\
\hline Median (IQR) & $62(5 I-73)$ & $63(52-73)$ & \\
\hline Minimum-maximum & $18-99$ & $18-100$ & \\
\hline \multicolumn{3}{|l|}{ Sex } & 0.93 \\
\hline Male & 3,501 (53.9\%) & 5,75 I (54.0\%) & \\
\hline Female & $2,992(46.1 \%)$ & $4,90 \mathrm{I}(46.0 \%)$ & \\
\hline \multicolumn{3}{|l|}{ Race/ethnicity } & $<0.001$ \\
\hline White & $2,938(45.3 \%)$ & $4,602(43.2 \%)$ & \\
\hline Black & $2,178(33.5 \%)$ & $3,365(31.6 \%)$ & \\
\hline Hispanic & $865(13.3 \%)$ & I,865 (I7.5\%) & \\
\hline Other & $512(7.9 \%)$ & $820(7.7 \%)$ & \\
\hline \multicolumn{3}{|l|}{ Dialysis vintage, months } & $<0.001$ \\
\hline 0 & 2,723 (4I.9\%) & $5,077(47.7 \%)$ & \\
\hline $1-12$ & I,037 (I6.0\%) & I,657 (I5.6\%) & \\
\hline$>12$ & $2,44 I(37.6 \%)$ & 3,449 (32.4\%) & \\
\hline Unknown & $292(4.5 \%)$ & $469(4.4 \%)$ & \\
\hline \multicolumn{3}{|l|}{ Primary cause of ESRD } & 0.02 \\
\hline Hypertension & $1,710(26.3 \%)$ & $3,012(28.3 \%)$ & \\
\hline Diabetes & 2,981 (45.9\%) & $4,785(44.9 \%)$ & \\
\hline Other/unknown & I,802 (27.8\%) & $2,855(26.8 \%)$ & \\
\hline Diabetes & $4,384(67.5 \%)$ & 7,202 (67.6\%) & 0.90 \\
\hline Heart failure & $1,285(19.8 \%)$ & $2,104(19.8 \%)$ & 0.95 \\
\hline Ischemic heart disease & $\mathrm{I}, 184(18.2 \%)$ & $2,006(18.8 \%)$ & 0.33 \\
\hline Cerebrovascular disease & $173(2.7 \%)$ & $266(2.5 \%)$ & 0.50 \\
\hline Peripheral vascular disease & $376(5.8 \%)$ & $596(5.6 \%)$ & 0.59 \\
\hline Cardiac dysrhythmia & $45 \mathrm{I}(7.0 \%)$ & $800(7.5 \%)$ & 0.17 \\
\hline COPD & $602(9.3 \%)$ & 949 (8.9\%) & 0.42 \\
\hline Liver disease & $123(1.9 \%)$ & $236(2.2 \%)$ & 0.15 \\
\hline Malignancy & $247(3.8 \%)$ & $417(3.9 \%)$ & 0.72 \\
\hline \multicolumn{3}{|l|}{ Charlson Comorbidity Index ${ }^{15}$} & 0.34 \\
\hline 2 & $495(7.6 \%)$ & 747 (7.0\%) & \\
\hline 3 & $486(7.5 \%)$ & $833(7.8 \%)$ & \\
\hline 4 & $931(14.3 \%)$ & $1,466(13.8 \%)$ & \\
\hline 5 & $1,156(17.8 \%)$ & I,825 (I7.1\%) & \\
\hline 6 & $1,201(18.5 \%)$ & $2,062(19.4 \%)$ & \\
\hline 7 & 991 (15.3\%) & $1,653(15.5 \%)$ & \\
\hline $8+$ & $1,233(19.0 \%)$ & $2,066(19.4 \%)$ & \\
\hline
\end{tabular}

Note: Tego needlefree connector is manufactured by ICU Medical, Inc., San Clemente, CA, USA.

Abbreviations: COPD, chronic obstructive pulmonary disease; ESRD, end-stage renal disease; IQR, interquartile range; SD, standard deviation.

Among controls, 2,225 IV antibiotic starts were identified in 1,694 patient-years at-risk time, and among Tego users, 3,308 IV antibiotic starts were identified in 2,811 patientyears at-risk time; this corresponded to crude rates of 131 and 118 events per 100-patient years for control and Tego patients, respectively. Tego use was associated with a $10 \%$ relative reduction in risk of IV antibiotic start (IRR $0.90,95 \%$ confidence interval $[\mathrm{CI}] 0.85-0.95 ; P<0.001)$. In a model adjusting for potential confounders that were imbalanced between exposure groups (model 1: adjusted for age, race, vintage,
Table 2 Comparison of catheter-related bloodstream infections in Tego and control cohorts

\begin{tabular}{|c|c|c|c|}
\hline & $\begin{array}{l}\text { Control } \\
(n=6,493)\end{array}$ & $\begin{array}{l}\text { Tego } \\
(n=10,652)\end{array}$ & $P$-value \\
\hline \multicolumn{4}{|l|}{ IV antibiotic starts } \\
\hline $\begin{array}{l}\text { At-risk time, patient- } \\
\text { years }\end{array}$ & 1,694 & 2,811 & \\
\hline Events & 2,225 & 3,308 & \\
\hline $\begin{array}{l}\text { Crude rate }(95 \% \mathrm{Cl}) \text {, } \\
\text { events per }\end{array}$ & $131(126-137)$ & $118(114-122)$ & \\
\hline 100 patient-years & & & \\
\hline Crude IRR $(95 \% \mathrm{Cl})$ & I (ref) & $0.90(0.85-0.95)$ & $<0.001$ \\
\hline $\begin{array}{l}\text { Model I: adjusted } \\
\text { IRR }(95 \% \mathrm{Cl})\end{array}$ & I (ref) & $0.92(0.87-0.97)$ & 0.002 \\
\hline $\begin{array}{l}\text { Model 2: adjusted } \\
\text { IRR }(95 \% \mathrm{Cl})\end{array}$ & I (ref) & $0.92(0.87-0.97)$ & 0.002 \\
\hline \multicolumn{4}{|c|}{ IV antibiotic course (2+ doses within 2 I-day period) } \\
\hline $\begin{array}{l}\text { At-risk time, } \\
\text { patient-years }\end{array}$ & 1,694 & 2,811 & \\
\hline Events & 1,730 & 2,499 & \\
\hline $\begin{array}{l}\text { Crude rate }(95 \% \mathrm{Cl}) \text {, } \\
\text { events per } \\
100 \text { patient-years }\end{array}$ & $102(97-107)$ & $89(85-92)$ & \\
\hline Crude IRR $(95 \% \mathrm{Cl})$ & I (ref) & $0.87(0.82-0.93)$ & $<0.001$ \\
\hline $\begin{array}{l}\text { Model I: adjusted } \\
\text { IRR }(95 \% \mathrm{Cl})\end{array}$ & I (ref) & $0.89(0.84-0.95)$ & $<0.001$ \\
\hline $\begin{array}{l}\text { Model 2: adjusted } \\
\text { IRR }(95 \% \mathrm{CI})\end{array}$ & I (ref) & $0.89(0.84-0.95)$ & $<0.001$ \\
\hline \multicolumn{4}{|l|}{ Positive blood culture } \\
\hline $\begin{array}{l}\text { At-risk time, } \\
\text { patient-years }\end{array}$ & 1,694 & 2,811 & \\
\hline Events & 526 & 828 & \\
\hline $\begin{array}{l}\text { Crude rate }(95 \% \mathrm{Cl}) \text {, } \\
\text { events per } \\
\text { I00 patient-years }\end{array}$ & $31(28-34)$ & $30(28-32)$ & \\
\hline Crude IRR $(95 \% \mathrm{Cl})$ & I (ref) & $0.95(0.85-1.06)$ & 0.34 \\
\hline $\begin{array}{l}\text { Model I: adjusted } \\
\text { IRR }(95 \% \mathrm{Cl})\end{array}$ & I (ref) & $0.98(0.88-1.09)$ & 0.72 \\
\hline $\begin{array}{l}\text { Model 2: adjusted } \\
\text { IRR }(95 \% \mathrm{Cl})\end{array}$ & I (ref) & $0.98(0.88-1.09)$ & 0.71 \\
\hline
\end{tabular}

Notes: Model I adjusted for age, race, vintage, and etiology in end-stage renal disease; Model 2 adjusted for all variables (age, sex, race, vintage, primary cause of ESRD, diabetes, heart failure, ischemic disease, cerebrovascular disease, cardiac dysrythmia, COPD, liver disease, malignancy, and Charlson comorbidity index). Tego needlefree connector is manufactured by ICU Medical, Inc., San Clemente, CA, USA.

Abbreviations: $\mathrm{Cl}$, confidence interval; IRR, incidence-rate ratio; IV, intravenous; ref, reference.

and etiology of ESRD) the protective association of Tego was slightly attenuated to relative reduction of $8 \%$ (IRR $0.92,95 \%$ CI 0.87-0.97), but was still statistically significant $(P=0.02)$. A fully adjusted model (model 2: adjusted for all covariates presented in Table 1) yielded estimates identical to those for model 1. Tego use was also associated with reduced risk of CRBSI, as assessed by receipt of an antibiotic course (unadjusted IRR $0.87,95 \%$ CI $0.82-0.93 ; P<0.001)$. Adjusting for covariates gave an IRR for Tego versus controls of $0.89(95 \%$ CI $0.84-0.95, P<0.001)$ for both model 1 and model 2. 

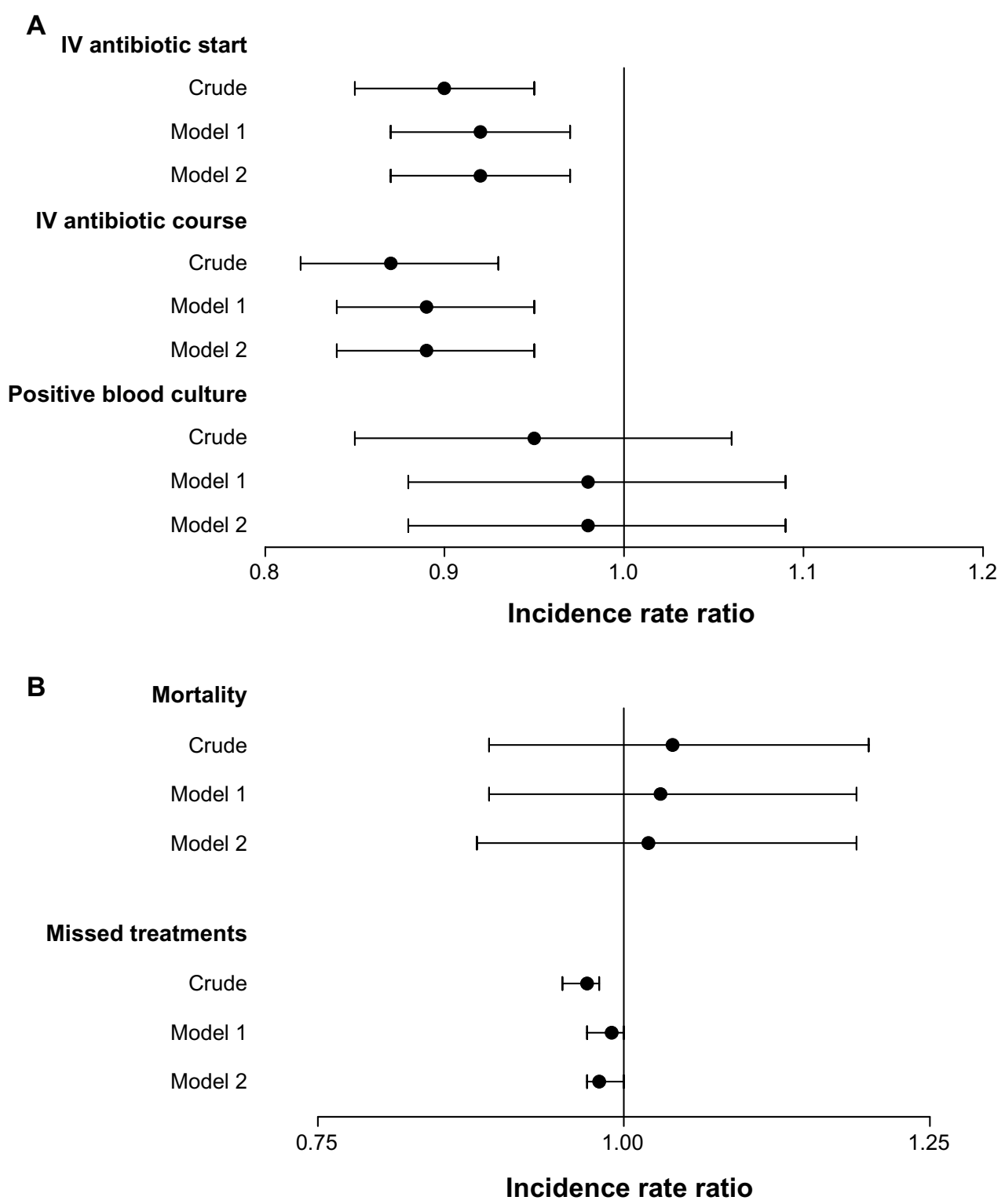

Figure I Associations between Tego use and catheter-related bloodstream infection (CRBSI), mortality, and missed dialysis treatments. Incidence-rate ratios (IRRs) with $95 \%$ confidence intervals are shown for (A) metrics of CRBSI and (B) mortality and missed dialysis treatments. IRRs for Tego versus controls are shown unadjusted (crude); adjusted for age, race, vintage, and etiology of ESRD (model I); model 2 adjusted for all variables (age, sex, race, vintage, primary cause of ESRD, diabetes, heart failure, ischemic disease, cerebrovascular disease, cardiac dysrythmia, COPD, liver disease, malignancy, and Charlson comorbidity index).

Note: Tego needlefree connector is manufactured by ICU Medical, Inc., San Clemente, CA, USA.

Abbreviations: IV, intravenous; ESRD, end-stage renal disease.

The unadjusted rate of positive blood cultures was 30 per 100 patient-years for Tego patients and 31 per 100 patient-years for controls. The unadjusted IRR for Tego patients versus controls was 0.95 (95\% CI 0.85-1.06, $P=0.34$ ); adjusted models showed no statistically significant differences between the cohorts.

\section{Mortality}

In the control cohort, 283 deaths occurred over 1,639 patientyears at-risk time, corresponding to a crude mortality rate of 17.3 deaths per 100 patient-years. In the Tego cohort, 486 deaths occurred over 2,719 patient-years at-risk time, corresponding to a crude mortality rate of 17.9 deaths per 100 patient-years. There was no significant association between Tego use and mortality rate observed on either unadjusted or adjusted analysis (Table 3 and Figure 1B).

\section{Missed dialysis sessions}

Tego patients missed fewer dialysis sessions than controls (17.2 versus 17.8 missed sessions per 100 patient-years). The unadjusted IRR for missed treatments (Tego patients 
Table 3 Comparison of mortality rates in Tego and control cohorts

\begin{tabular}{|c|c|c|c|}
\hline & $\begin{array}{l}\text { Control } \\
(n=6,493)\end{array}$ & $\begin{array}{l}\text { Tego } \\
(n=10,652)\end{array}$ & $P$-value \\
\hline At-risk time, pt-years & I,639 & 2,719 & \\
\hline Deaths & 283 & 486 & \\
\hline $\begin{array}{l}\text { Crude mortality rate } \\
(95 \% \mathrm{Cl}) \text {, deaths per } \\
100 \text { pt-years }\end{array}$ & $17.3(15.3-19.4)$ & $17.9(16.3-19.5)$ & \\
\hline Crude IRR $(95 \% \mathrm{Cl})$ & I (ref) & I.04 (0.89-I.20) & 0.64 \\
\hline $\begin{array}{l}\text { Model I: adjusted } \\
\text { IRR }(95 \% \mathrm{Cl})\end{array}$ & I (ref) & $1.03(0.89-1.19)$ & 0.71 \\
\hline $\begin{array}{l}\text { Model 2: adjusted } \\
\text { IRR }(95 \% \mathrm{Cl})\end{array}$ & I (ref) & $1.02(0.88-1.19)$ & 0.74 \\
\hline
\end{tabular}

Notes: Model I adjusted for age, race, vintage and etiology in end-stage renal disease; model 2 adjusted for all variables (age, sex, race, vintage, primary cause of ESRD, diabetes, heart failure, ischemic disease, cerebrovascular disease, cardiac dysrythmia, COPD, liver disease, malignancy, and Charlson comorbidity index). Tego needlefree connector is manufactured by ICU Medical, Inc., San Clemente, CA, USA.

Abbreviations: $\mathrm{Cl}$, confidence interval; IRR, incidence-rate ratio; pt, patient; ref, reference.

relative to controls) was 0.97 (95\% CI 0.95-0.98; Table 4 and Figure 1B). In fully adjusted analyses, differences were attenuated, but remained statistically significant (model 2, IRR 0.98, $P=0.04)$.

\section{Medication use}

Figure 2 shows the probability of patients receiving ESAs and the per-session ESA dose among users for each cohort in each month of the study: Figure $2 \mathrm{~A}$ and $\mathrm{C}$ present unadjusted estimates, and Figure $2 \mathrm{~B}$ and $\mathrm{D}$ show adjusted estimates (model 1: adjusted for age, race, vintage, and etiology of

Table 4 Comparison of missed dialysis treatments in Tego and control cohorts

\begin{tabular}{|c|c|c|c|}
\hline & $\begin{array}{l}\text { Control } \\
(n=6,493)\end{array}$ & $\begin{array}{l}\text { Tego } \\
(n=10,652)\end{array}$ & P-value \\
\hline At-risk time, pt-years & 1,639 & 2,719 & \\
\hline Missed treatments & 29,153 & 46,749 & \\
\hline $\begin{array}{l}\text { Crude missed-treatment } \\
\text { rate }(95 \% \mathrm{Cl}) \text {, missed } \\
\text { treatments per } \\
100 \text { pt-years }\end{array}$ & I7.8 (17.6-18.0) & I7.2 (I7.0-17.4) & \\
\hline Crude IRR $(95 \% \mathrm{CI})$ & I (ref) & $0.97(0.95-0.98)$ & $<0.001$ \\
\hline $\begin{array}{l}\text { Model I: adjusted } \\
\text { IRR }(95 \% \mathrm{Cl})\end{array}$ & I (ref) & $0.99(0.97-1.00)$ & 0.11 \\
\hline $\begin{array}{l}\text { Model 2: adjusted } \\
\text { IRR }(95 \% \mathrm{Cl})\end{array}$ & I (ref) & $0.98(0.97-1.00)$ & 0.04 \\
\hline
\end{tabular}

Notes: Model I adjusted for age, race, vintage and etiology in end-stage renal disease; model 2 adjusted for all variables (age, sex, race, vintage, primary cause of ESRD, diabetes, heart failure, ischemic disease, cerebrovascular disease, cardiac dysrythmia, COPD, liver disease, malignancy, and Charlson comorbidity index). Tego needlefree connector is manufactured by ICU Medical, Inc., San Clemente, CA, USA.

Abbreviations: $\mathrm{Cl}$, confidence interval; IRR, incidence-rate ratio; $\mathrm{pt}$, patient; ref, reference.
ESRD). Both the probability of receiving ESAs and the persession dose declined over the course of the study for both groups. The adjusted probability of receiving ESAs was similar between the two groups at all times, except in months 4 (adjusted model only) and 6 (unadjusted and adjusted models), where use among Tego patients was modestly higher. Mean per-session ESA doses for the Tego and control cohorts declined month on month until month 6 . Thereafter, while the decline in per-session dose continued for Tego patients, the per-session dose began to increase for control patients, resulting in statistically significant differences between the cohorts being observed in months $7(P=0.02), 8(P<0.001)$, and $9(P=0.009)$.

The unadjusted per-month probability of receiving thrombolytic treatment was $6.2 \%$ for control patients compared to $5.6 \%$ for Tego patients ( $P=0.007$; Figure $2 \mathrm{E}$ ). Adjusting for potential confounders (age, race, vintage, etiology of ESRD) yielded similar results $(6.3 \%$ versus $5.6 \%$ for controls versus Tego, $P=0.03)$.

\section{Discussion}

In this retrospective analysis of 17,145 patients receiving incenter HD, Tego connector use was found to be independently associated with a $10 \%-12 \%$ reduction in risk of CRBSIs, as defined by initiation of IV antibiotics or initiation of an IV antibiotic course. A directionally similar, although not statistically significant, association was observed when CRBSIs were defined by positive blood culture. It should be noted, however, that the lack of statistical significance for this indicator of infection may be due at least in part to a paucity of identified events: many patients receive blood cultures in the context of hospitalization or emergency care, and such cultures were not captured in the data assessed. The fact that similar reductions in the risk of CRBSI were identified using all three definitions suggests that the observed associations were unlikely due to chance or to choice of definition of infection.

A previously published quality improvement initiative involving a small number of pediatric HD patients also demonstrated a decrease in catheter infections upon switching to Tego connectors locked with heparin, ${ }^{14}$ while a more recent randomized controlled trial in 66 adult HD patients at a single center showed no difference in incidence of bacteremia with Tego connectors locked with saline compared to citratelocked standard catheter caps. ${ }^{13}$ These studies are limited, however, by their small size, and were likely underpowered. The current analysis represents, to our knowledge, the first large-scale evaluation of the Tego connector in a real-world HD population. 
A

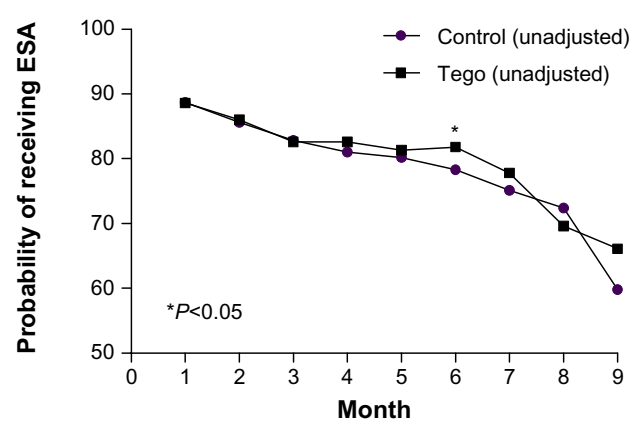

C

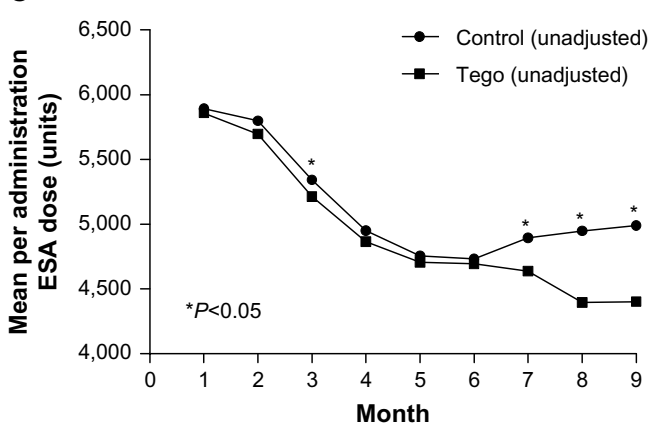

B

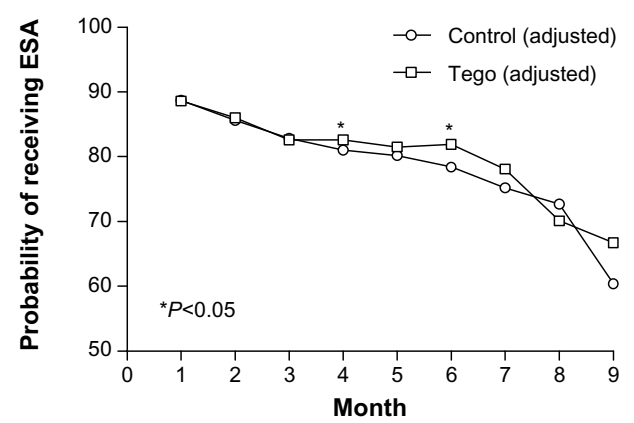

D

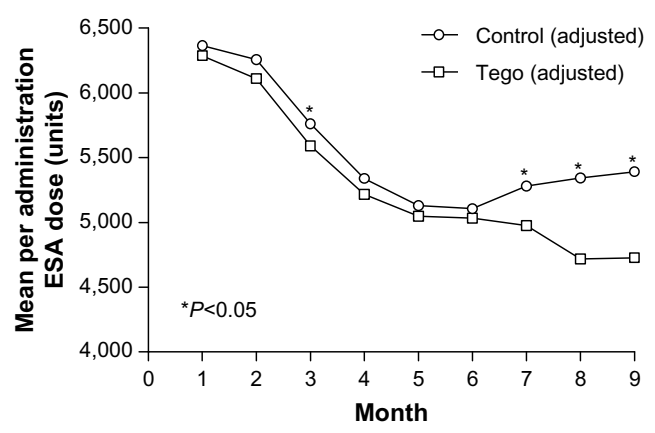



Figure 2 (A-E) Medication utilization for Tego and control cohorts. The probability of receiving an erythropoiesis-stimulating agent (ESA) and the per-session ESA dose among users for Tego and control patients in each month of the study are shown. (A and C) Unadjusted estimates; (B and D) estimates adjusted for age, race, vintage, and etiology of end-stage renal disease (model I). Unadjusted and adjusted (model I) estimates of the per-month probability of thrombolytic use over the entire study period are shown in panel $\mathrm{E}$.

Notes: *Months in which the difference between Tego and control groups was statistically significant $(P<0.05)$. Tego needlefree connector is manufactured by ICU Medical, Inc., San Clemente, CA, USA.

Beyond the association with reduced incidence of CRBSIs, Tego use was found to be independently associated with a decreased rate of missed dialysis treatments, corresponding to approximately 0.6 fewer missed treatments per patient per year. Patients who frequently miss dialysis sessions have higher rates of morbidity and mortality than those who attend according to their prescribed schedule, and thus a reduction in the number of missed sessions may have clinical benefits. Moreover, even a small reduction in the frequency of missed treatments provides cost benefits to dialysis providers.

Unlike conventional catheter caps, the Tego connector can be flushed and locked with saline, reducing the need for heparin or other antithrombotic locking solutions. ${ }^{12,13}$ A potential concern, however, is that use of the Tego connector locked with saline may lead to increased risk of catheter occlusions. Our data do not support this hypothesis, and point estimates show that thrombolytic use (a marker of thrombotic complications) was modestly decreased with Tego use, although it should be noted that thrombolytic usage overall was very low. Moreover, it is important to note that we lacked data on interventional procedures (eg, catheter exchanges), and therefore cannot make definitive conclusions about relative performance with respect to mechanical catheter complications.

There was a downward trend in ESA use over the study for both Tego and control patients. This is perhaps to be expected, as it has been reported that ESA doses are highest in incident 
patients (with a peak at 2 months after onset of dialysis), declining over time as hemoglobin levels normalize, ${ }^{1}$ and 40\%-50\% of patients in this study were new to dialysis at the study start. In addition to the overall downward trend, Tego use was associated with lower ESA utilization compared to controls in months 7-9 following catheter placement. Per-administration dose differences of approximately 500 Units were observed at later time points in the study, which could have a substantive financial impact for dialysis providers, particularly in the light of the recent rebasing of Centers for Medicare and Medicaid Services reimbursement rates for dialysis. ${ }^{16}$

One plausible hypothesis is that ESA requirements might be lower among Tego patients by virtue of lower catheter infection/colonization burden. The delayed separation in ESA utilization may reflect the obligate time needed for sufficient numbers of patients to develop meaningful levels of bacterial colonization, as well as response time in titrating ESAs. Considered alongside antibiotic data, we interpret these data to enrich our inference on vascular-related infections (both overt and subclinical). No significant association between Tego use and mortality was observed. However, we acknowledge that given the relatively short duration of the study, follow-up may not have been long enough to meaningfully assess mortality risk.

As for all observational studies, only associations between exposure and outcomes could be assessed; a randomized trial would be needed to demonstrate causality. Although models were adjusted for covariates that differed between the control and Tego cohorts, the possibility of residual confounders cannot be excluded.

Infectious complications due to chronic catheter use in HD patients who are not good candidates for arteriovenous fistulas or arteriovenous grafts are an ongoing concern. Our findings suggest that the use of the Tego connector may reduce the risk of CRBSIs, and in addition may result in lower utilization of thrombolytics, antibiotics, and ESAs, as well as fewer missed dialysis treatments; however, a randomized controlled trial would be needed to confirm these findings. Budget impact models accounting for all of these factors, as well as the cost of the device itself, will be required to quantify the potential cost benefit of the Tego connector to payers.

\section{Acknowledgment}

We thank DaVita Clinical Research ${ }^{\circledR}\left(\mathrm{DCR}^{\circledR}\right)$ for providing technical support for this research project. DCR is committed to advancing the knowledge and practice of kidney care.

\section{Disclosure}

This study was supported by ICU Medical, Inc. SMB is an employee of DCR, and has served on advisory boards for Keryx Biopharmaceuticals, Otsuka Pharmaceutical, Amgen, and CB Fleet; SMB's spouse is employed by AstraZeneca. LN is an employee of DaVita HealthCare Partners, Inc. AEH is an employee of DCR. SPS is an employee of DCR, and owns stock in DaVita HealthCare Partners, Inc.

\section{References}

1. United States Renal Data System. 2013 USRDS Annual Data Report: Atlas of End-Stage Renal Disease in the United States. Minneapolis: USRDS; 2013. http://www.usrds.org/atlas.aspx. Accessed November 20, 2013.

2. Chawla LS, Krishnan M. Causes and consequences of inflammation on anemia management in hemodialysis patients. Hemodial Int. 2009;13(2):222-234.

3. Movilli E, Brunori G, Camerini C, et al. The kind of vascular access influences the baseline inflammatory status and epoetin response in chronic hemodialysis patients. Blood Purif. 2006;24(4):387-393.

4. McGee DC, Gould MK. Preventing complications of central venous catheterization. N Engl J Med. 2003;348(12):1123-1133.

5. Kadidal VV, Mayo DJ, Horne MK. Heparin-induced thrombocytopenia (HIT) due to heparin flushes: a report of three cases. J Intern Med. 1999;246(3):325-329.

6. Moran JE, Ash SR. Locking solutions for hemodialysis catheters; heparin and citrate - a position paper by ASDIN. Semin Dial. 2008;21(5):490-492.

7. Shanks RM, Donegan NP, Graber ML, et al. Heparin stimulates Staphylococcus aureus biofilm formation. Infect Immun. 2005;73(8): 4596-4606.

8. Shanks RM, Sargent JL, Martinez RM, Graber ML, O’Toole GA. Catheter lock solutions influence staphylococcal biofilm formation on abiotic surfaces. Nephrol Dial Transplant. 2006;21(8): $2247-2255$.

9. Lacson E Jr, Wang W, DeVries C, et al. Effects of a nationwide predialysis educational program on modality choice, vascular access, and patient outcomes. Am J Kidney Dis. 2011;58(2):235-242.

10. Wilson SM, Mayne TJ, Krishnan M, et al. CathAway fistula vascular access program achieves improved outcomes and sets a new standard of treatment for end-stage renal disease. Hemodial Int. 2013;17(1): 86-93.

11. Lacson E Jr, Lazarus JM, Himmelfarb J, Ikizler TA, Hakim RM. Balancing fistula first with catheters last. Am J Kidney Dis. 2007; 50(3):379-395.

12. ICU Medical. Tego needlefree connector. Available from: http://www. icumed.com/products/specialty/renal-systems/tego-connector.aspx. Accessed November 20, 2013.

13. Bonkain F, Racapé J, Goncalvez I, et al. Prevention of tunneled cuffed hemodialysis catheter-related dysfunction and bacteremia by a neutral-valve closed-system connector: a single-center randomized controlled trial. Am J Kidney Dis. 2013;61(3):459-465.

14. McAfee N, Seidel K, Watkins S, Flynn JT. A continuous quality improvement project to decrease hemodialysis catheter infections in pediatric patients: use of a closed luer-lock access cap. Nephrol Nurs J. 2010;37(5):541-544; quiz 545.

15. Charlson ME, Pompei P, Ales KL, MacKenzie CR. A new method of classifying prognostic comorbidity in longitudinal studies: development and validation. J Chronic Dis. 1987;40(5):373-383.

16. Centers for Medicare and Medicaid Services. End-stage renal disease prospective payment system and quality incentive program. Final rule. Fed Regist. 2013;78(231):72156-72251. 


\section{Publish your work in this journal}

The International Journal of Nephrology and Renovascular Disease is an international, peer-reviewed open-access journal focusing on the pathophysiology of the kidney and vascular supply. Epidemiology, screening, diagnosis, and treatment interventions are covered as well as basic science, biochemical and immunological studies. The journal welcomes original research, clinical studies, reviews \& evaluations, expert opinion and commentary, case reports and extended reports. The manuscript management system is completely online and includes a very quick and fair peerreview system, which is all easy to use. Visit http://www.dovepress.com/ testimonials.php to read real quotes from published authors

Submit your manuscript here: http://www.dovepress.com/international-journal-of-nephrology-and-renovascular-disease-journal 